\title{
Lames à usage unique et lames réutilisables: étude randomisée contrôlée en chirurgie réglée
}

\section{Single-use and reusable laryngoscope blades: a randomized controlled trial in elective surgery}

\author{
Philippe Guerci, MD • Florence Vial, MD • \\ Hervé Bouaziz, MD, PhD
}

Received: 2 August 2011/Accepted: 23 September 2011/Published online: 14 October 2011

(C) Canadian Anesthesiologists' Society 2011

Au rédacteur en chef,

Les nouvelles normes d'hygiène liées à l'émergence des agents pathogènes transmissibles non conventionnels ont conduit à l'utilisation de lames de laryngoscopie à usage unique (UU). On a démontré la supériorité ${ }^{1}$ ou la non-infériorité ${ }^{2}$ des lames métalliques UU lors d'inductions en séquence rapide. Le but de ce travail était de comparer en chirurgie réglée la qualité de visualisation glottique lors de l'utilisation successive de lames métalliques réutilisables (R) et de lames UU.

Il s'agit d'une étude prospective, randomisée et contrôlée, monocentrique. Après accord du comité de protection des personnes, 100 patientes subissant une chirurgie abdominopelvienne réglée ont été randomisées en deux groupes : Groupe UU-R (première laryngoscopie avec la lame UU puis la lame R), Groupe R-UU (première laryngoscopie avec la lame $\mathrm{R}$ puis la lame $\mathrm{UU}$ ). Toutes les patientes ont bénéficié d'un protocole anesthésique standardisé comprenant une anesthésie intraveineuse à objectif de concentration titrée sur l'index bispectral (BIS) et curarisation. Le critère de jugement principal était l'incidence des scores de Cormack et Lehane (CL) simples (1-2) et difficiles (3-4), après chaque laryngoscopie. Les critères secondaires étaient le pourcentage d'ouverture glottique (POGO), la satisfaction de l'opérateur, la survenue de difficultés d'intubation et les moyens utilisés.

L'incidence des laryngoscopies difficiles (CL 3 ou 4) était de $11 \%$ pour les lames UU et $9 \%$ pour les lames R $(P=0,16)$ (Tableau). Le score de POGO était le même pour les deux lames (Tableau). Une mobilisation cervicale était nécessaire dans 11 cas (UU) contre $16(\mathrm{R})(P=0,26)$.

P. Guerci, MD $(\bowtie) \cdot$ F. Vial, MD · H. Bouaziz, MD, PhD

Maternité Régionale de Nancy, Nancy, France

e-mail: phil.guerci@gmail.fr
Aucun échec d'intubation n'était noté avec la lame UU contre trois avec la lame $\mathrm{R}(P=0,07)$. Ces trois patientes ont été intubées grâce à l'utilisation d'un mandrin d'Eschmann. La satisfaction des opérateurs pour l'une ou l'autre lame n'était pas différente $(P=0,16)$.

Cette étude ne met pas en évidence de supériorité des lames métalliques à usage unique sur les lames métalliques réutilisables en termes de visualisation glottique. L'évaluation de ce critère est en accord avec les données de la littérature lors d'inductions en séquence rapide, ${ }^{1,2}$ à l'exception de l'incidence plus faible des CL 3 pour les lames UU dans l'étude d'Amour et al. ${ }^{1}$ L'incidence des échecs d'intubation n'est pas significativement différente même si une tendance favorable aux lames à UU a été constatée. Une tendance favorable aux lames UU est également observée sur mannequin dans des conditions d'intubation difficile, contrairement aux études réalisées chez les patients lors d'induction en séquence rapide. ${ }^{1-3}$ L'absence de différence sur le critère d'échec d'intubation s'explique vraisemblablement par un manque de puissance de la présente étude.

Différents points nécessitent d'être soulevés concernant les limites de cette étude. L'évaluation de la visualisation glottique fait partie des critères de prédiction d'une intubation difficile. Elle ne permet cependant pas à elle seule de prédire toutes les difficultés possibles. D'autres critères doivent lui être associés. Le choix du score de CL comme critère d'évaluation présente des limites. Très utilisé, son appréciation par des anesthésistes expérimentés reste aléatoire. ${ }^{4}$ Le score de POGO est un critère d'évaluation plus sensible de la visualisation glottique. ${ }^{5}$ Il fournit une meilleure fiabilité inter et intra observateur. Aucune différence significative de POGO n'a été objectivée entre les deux groupes. Cependant, l'évaluation des scores de CL et du score de POGO permettait de comparer les deux 
Tableau Évaluation des scores Cormack et Lehane, et score de POGO

$\begin{array}{lll}\text { Lame réutilisable } & \text { Lame à usage } & P \\ n=100 & \text { unique } n=100\end{array}$

Score Cormack et Lehane (Nombre de patientes)

\begin{tabular}{clll} 
Grade 1 ou 2 & 91 & 89 & 0,16 \\
Grade 3 ou 4 & 9 & 11 & 0,16 \\
Score de POGO $(\%)$ & $80[60-90]$ & $80[60-90]$ & 0,27 \\
$\begin{array}{c}\text { Score de satisfaction à } \\
\text { 3 ou } 4(\%)\end{array}$ & 94 & 96 & 0,16 \\
\hline
\end{tabular}

Les valeurs de POGO sont exprimées en médianes et interquartiles $[25 \%-75 \%]$. POGO $=$ pourcentage d'ouverture glottique

lames chez un même patient. La prise du sujet comme son propre témoin diminue la variabilité de mesure et améliore la fiabilité d'autant que les conditions de visualisation aux deux temps étaient optimisées. L'avantage de l'essai croisé est d'être plus puissant que l'essai en bras parallèles, à effectif identique.

En conclusion, les lames métalliques UU alliant sécurité microbiologique, et efficacité technique apparaissent en chirurgie réglée, comme un matériel performant pour l'intubation orotrachéale.

Conflits d'intérêt Aucun.

\section{Références}

1. Amour J, Le Manach YL, Borel M, et al. Comparison of single-use and reusable metal laryngoscope blades for orotracheal intubation during rapid sequence induction of anesthesia: a multicenter cluster randomized study. Anesthesiology 2010; 112: 325-32.

2. Jabre P, Galinski M, Ricard-Hibon A, et al. Out-of-hospital tracheal intubation with single-use versus reusable metal laryngoscope blades: a multicenter randomized controlled trial. Ann Emerg Med 2011; 57: 225-31.

3. Twigg SJ, McCormick B, Cook TM. Randomized evaluation of the performance of single-use laryngoscopes in simulated easy and difficult intubation. Br J Anaesth 2003; 90: 8-13.

4. Krage $R$, van Rijn $C$, van Groeningen D, Loer SA, Schwarte LA, Schober P. Cormack-Lehane classification revisited. Br J Anaesth 2010; 105: 220-7.

5. Ochroch EA, Hollander JE, Kush S, Shofer FS, Levitan RM. Assessment of laryngeal view: percentage of glottic opening score vs Cormack and Lehane grading. Can J Anesth 1999; 46: 987-90. 